\title{
Permeability of Porous Poly(3-hydroxybutyrate) Barriers of Single and Bilayer Type for Implant Applications
}

\author{
Anna Bergstrand, ${ }^{1,2}$ Sanna Uppström, ${ }^{1}$ and Anette Larsson ${ }^{1,2}$ \\ ${ }^{1}$ Department of Chemical and Biological Engineering, Chalmers University of Technology, \\ 41296 Gothenburg, Sweden \\ ${ }^{2}$ SuMo Biomaterials, VINN Excellence Center, 41296 Gothenburg, Sweden \\ Correspondence should be addressed to Anna Bergstrand; anna.bergstrand@chalmers.se
}

Received 1 July 2013; Revised 22 September 2013; Accepted 6 October 2013; Published 2 January 2014

Academic Editor: Haojun Liang

Copyright (C) 2014 Anna Bergstrand et al. This is an open access article distributed under the Creative Commons Attribution License, which permits unrestricted use, distribution, and reproduction in any medium, provided the original work is properly cited.

Poly(3-hydroxybutyrate) (PHB) is a polyester which shows excellent biocompatibility and a PHB material is therefore considered suitable for many biomedical applications. A highly porous PHB material may be designed to facilitate the transport of small molecules and body fluids or serve as a biocompatible temporary barrier. In this study, PHB films with varying degree of porosity and pore interconnectivity were made by solvent casting using water-in-oil emulsion templates of varying composition. The morphology was characterized by SEM and the water permeability of the films was determined. The results show that an increased water content of the template emulsion resulted in a film with increased porosity. A fine tuning of the film morphology of the casted films was achieved by varying the salt content of the water phase of the template emulsion. The porosity of these films was roughly the same but the water permeability varied between $23 \cdot 10^{-13}$ and $1486 \cdot 10^{-13} \mathrm{~m}^{2} / \mathrm{s}$. It was concluded that the major determinant of the water permeability through these films is the pore interconnectivity. Furthermore, we report on the formation and water permeability of bilayer PHB films consisting of a porous layer combined with a dense backing layer.

\section{Introduction}

Poly(3-hydroxybutyrate) (PHB) is considered to be a polymer that has high potential as a biodegradable implant material. The polymer is a well examined thermoplastic polyester of microbial origin which shows excellent biocompatibility in contact with tissue and blood [1-5]. The natural presence of low molecular weight $\mathrm{PHB}$ and the polymer degradation product, 3-hydroxybutyric acid, in the body is further evidence for the nontoxicity of PHB [6]. Besides the necessary biocompatibility of $\mathrm{PHB}$, the use as an implant requires that the material is degraded during a period of time that is relevant for the application in question. $\mathrm{PHB}$ is one of the slowest degrading polyesters since the polymer is highly crystalline, preventing water penetration into the polymer matrix and subsequent scission of polymer chains [7]. An implant made out of $\mathrm{PHB}$ material can retain its integrity during an extended period of time in a wet environment and this feature may be attractive for certain applications. PHB has been investigated for many types of medical applications including gastrointestinal patches [8], nerve guides $[9,10]$, and scaffolds for tissue engineering [11].

The fabrication of highly porous materials with interconnected pores is of great interest in the field of tissue engineering since it enables the passage of fluid and transport of small molecules such as nutrients, drugs, or bioactive molecules to soft tissue and cells. If the material is made macroporous then cells may interpenetrate via the pore structures. However, if the material is made microporous (pore sizes of a few micrometers and below) such a material has the potential to serve as a biocompatible temporary barrier preventing cellular ingrowth yet facilitating transport to adjacent soft tissue and cells. Moreover, by combining a porous and a dense backing layer, new 
permeability and degradation properties are acquired which opens up for additional applications such as directional transport of small bioactive molecules or side directed cell growth.

Currently there are several different techniques such as salt leaching [12-14], phase separation [15], or structuring techniques $[16,17]$ to produce porous biodegradable materials. The pore size varies, but generally these techniques result in materials with macroporous structures of sizes from 10 to $500 \mu \mathrm{m}$ and they include several preparation steps which make them labor intensive and time-consuming. In a former study, we reported on the use of water-in-oil emulsion template in combination with solvent casting to fabricate microporous PHB foams $[18,19]$. Here we further elaborated on this concept by producing porous $\mathrm{PHB}$ films with varying degree of pore interconnectivity. This was done by changing the composition of the emulsion template. The effect of increasing the water fraction or salt concentration of the template emulsion on film porosity and pore interconnectivity was studied and the permeability was determined. It was further tested if a dense film layer could successfully be combined with a porous layer. A measure of success was if the bilayer film acquired equivalent permeability as the dense single layer film.

\section{Materials and Methods}

2.1. Materials. Poly-[(R)-3-hydroxybutyrate], PHB, (natural origin), chloroform (purity 99.0-99.4\%, stabilized with $1 \%$ ethanol), sorbitan monooleate (Span 80), and lithium sulphate monohydrate were purchased from Sigma-Aldrich, (Germany). Milli-Q water was used for the emulsion preparation. Ultima gold and tritiated water were purchased from PerkinElmer (USA). All chemicals were used as received.

\subsection{Preparation of PHB Solution and Template Emulsions.} Water-in-oil (w/o) template emulsions were prepared from a $7 \%(\mathrm{w} / \mathrm{v})$ PHB solution and a water phase consisting of lithium sulphate salt and Milli-Q water. A range of template emulsions was produced by varying the amount of water $(0-10 \%(\mathrm{v} / \mathrm{v}))$ and lithium sulphate $(0-14.3 \%(\mathrm{w} / \mathrm{v}))$ of the emulsion. In a typical procedure making the emulsion, $560 \mathrm{mg} \mathrm{PHB}$ was dissolved in $8 \mathrm{~mL}$ of chloroform at $58^{\circ} \mathrm{C}$, under vigorous stirring with a magnetic bar for 30-45 minutes until a clear solution was obtained. The polymer solution was cooled to room temperature before addition of $100 \mu \mathrm{L}$ of $1.5 \%$ (w/v) Span80 in chloroform followed by stirring for a few minutes. An aqueous solution of $2.9 \%(\mathrm{w} / \mathrm{v})$ $\mathrm{Li}_{2} \mathrm{SO}_{4}$ in Milli-Q water was prepared. The emulsion was made by slowly injecting $800 \mu \mathrm{L}$ of the aqueous solution to the PHB solution with a Hamilton syringe during heavy stirring with a disperser (IKA DI18, Brazil) at $22000 \mathrm{rpm}$. The emulsion was subsequently homogenized for $5 \mathrm{~min}$ at $22000 \mathrm{rpm}$ on iced water. Pure PHB films were prepared from a $7 \%(\mathrm{w} / \mathrm{v}) \mathrm{PHB}$ solution by dissolving $560 \mathrm{mg} \mathrm{PHB}$ in $8 \mathrm{~mL}$ chloroform at $58^{\circ} \mathrm{C}$, during vigorous stirring with a magnetic bar for 30-45 minutes until a clear solution was obtained.
2.3. Preparation of Single Layer Films from Template Emulsions and Pure PHB Solutions. Films were prepared by solvent casting of PHB solutions into glass Petri dishes. Approximately $7 \mathrm{~mL}$ of the prepared emulsions or pure PHB solutions was poured into Petri dishes with an inner diameter of $7 \mathrm{~cm}$. Tryouts were made to establish the optimal drying conditions and solvent evaporation rate that gave the best quality of the resulting film. Typically, the PHB solution was poured out and preconcentrated by solvent evaporation from the emulsion for two minutes with the lid open on the dish. Subsequently, the Petri dish was covered with a glass lid leaving an opening of about $1 \mathrm{~mm}$ between the dish and lid during film formation. The films were left to dry for about 36 hours at $22^{\circ} \mathrm{C}$ in a fume cupboard and subsequently in a vacuum oven, at $40^{\circ} \mathrm{C}$ for 24 hours, in order to evaporate the remains of chloroform. The film thickness films was measured by a micrometer gauge (Mitutoyo, Japan) and determined from an average of the thickness at five positions of each sample.

2.4. Preparation of Bilayer PHB Films. The first layer was prepared from a solution of $2 \mathrm{~mL}$ of $3.5 \%(\mathrm{w} / \mathrm{v})$ PHB dissolved in chloroform at $58^{\circ} \mathrm{C}$, during vigorous stirring for $30-$ 45 minutes until a clear solution was obtained. The PHB solution was casted in a Petri dish forming a first film layer by preconcentrating the polymer solution for two minutes without lid, closing the lid for two minutes, and finally the lid was removed for two more minutes generating a sticky semidry film. A second layer was casted on top of the first PHB layer in the Petri dish by adding $4 \mathrm{~mL}$ of an emulsion consisting of $10 \%(\mathrm{v} / \mathrm{v})$ water, $7 \%(\mathrm{w} / \mathrm{v})$ PHB, $2.9 \%(\mathrm{w} / \mathrm{v})$ $\mathrm{Li}_{2} \mathrm{SO}_{4}$, and $1.5 \%(\mathrm{w} / \mathrm{w}) \mathrm{Span} 80$. The emulsion was prepared according to the method described in Section 2.2.

2.5. Scanning Electron Microscopy. The dry films were cut into sections, repeatedly submerged into liquid nitrogen for a few minutes, and freeze-fractured to display the interior regions of the film. The film specimens were sputter-coated with gold and visualized by in-lens and horizontal detection mode using a field emission scanning electron microscope (SEM) (Leo Ultra 55 FEG SEM) at a magnification of $5-30 \mathrm{k}$ at $3 \mathrm{kV}$.

2.6. Water Permeability Analysis. The water permeability of the films was determined using a permeability chamber with a setup previously described [20]. In short, a film sample was placed between a donor and an acceptor compartment. Initially, $15 \mathrm{~mL}$ of Milli-Q water was added simultaneously to both compartments. The temperature was maintained at $37^{\circ} \mathrm{C}$ through the analyses and two paddles were used to stir the contents of the two compartments at a speed of approximately $200 \mathrm{rpm}$. After three minutes, a small amount of tritiated water $(10 \mu \mathrm{L}, 400 \mathrm{kBq})$ was added to the donor compartment. At specified times, $500 \mu \mathrm{L}$ sample was taken from the acceptor compartment and was replaced by the same volume of Milli-Q water. The samples collected at the specified time points were weighed, mixed with Ultima gold 
at a 1/10 ratio, and analyzed in a scintillation counter (TriCarb 2810 TR, PerkinElmer, USA). The volume of water that had diffused through the film at each time point could be determined from the tritium activity registered in the acceptor compartment. The water permeability was subsequently determined according to assumptions and equations stated in Section 3.2 and normalized against the film thickness. The average permeability and $\mathrm{min} / \mathrm{max}$ deviation was determined from $n=2-3$ measurements.

2.7. Film Porosity. Circular samples of $5 \mathrm{~mm}$ in diameter were punched and weighed and the thickness measured by a micrometer gauge (Mitutoyo, Japan). The apparent film density was determined from the weight and dimensions of each of the porous PHB films $(n=3)$. The porosity was calculated by dividing the apparent film density with the true density of PHB (see (5)).

\section{Results and Discussion}

Template emulsions with adjustable amount of included water and lithium salt content were used for the fabrication of films with varying extent of porosity. Films were also made from a pure PHB solution to produce dense and nonporous PHB films. In addition, bilayer films were made by cocasting a dense PHB layer with a highly porous layer of PHB. The films were then characterized with respect to morphology, water permeability, and porosity.

\subsection{Film Morphology}

3.1.1. Single Layer Films. All films were visually inspected after the casting and drying procedure. The film cast from a PHB solution was semitransparent with a smooth upper surface. The PHB films casted from emulsion templates appeared increasingly whiter and less transparent with increasing amount of included water in the template emulsion. The upper surfaces appear smooth for all the films produced from emulsion templates at an ocular inspection. SEM was used to visualize the film morphology and disclose the pore structures and distribution at the surface and cross sections of the films made from emulsion templates and pure PHB films. The SEM images of cross sections from films made with $3-10 \%$ of water in the emulsion template and pure $\mathrm{PHB}$ are shown in Figure 1. It can be seen that the pure PHB film is dense and without pores. Further it can be seen that the films made from emulsion templates display a variation in porosity depending on the amount of water in the emulsion template. However they all show a homogeneous porous morphology with the pores evenly distributed over the cross section of the film.

The SEM images show that the films made from emulsion templates including 3 and 6\% water (Figures 1(b) and 1(c)) have pore structures which appear similar in character with small pores of about $0.5-1 \mu \mathrm{m}$ equally distributed in the film. The films made from emulsion templates including 8,9 , and $10 \%$ water (Figures $1(\mathrm{~d}), 1(\mathrm{e})$, and $1(\mathrm{f})$ ) have a higher amount of visible pores of about $1-3 \mu \mathrm{m}$ and the appearance of a solid foam. The film template with $10 \%$ water appears to have the most close packing of pores where adjacent pores seem to be connected through small openings, forming so called windows of about $0.5 \mu \mathrm{m}$ size. In order to study this in detail, SEM pictures were taken with in-lens detection mode at high magnification, $30 \mathrm{k}$, to disclose the pore architecture on the surface of this film. The SEM picture in Figure 2 shows a film made from a template emulsion including $10 \%$ water. It can be seen that the film is certainly highly porous with pore sizes of approximately $0.5-1 \mu \mathrm{m}$. The pores seem to be connected by windows, possibly forming interconnected pore routes or channels through the film. The extent of interconnection is difficult to comprehend. The channels may be partly discrete and discontinuous but they also have the potential to span the complete cross section of the film.

The results from the SEM experiments show that a high fraction of water in the template emulsion results in a highly porous PHB film. For the film with $10 \%$ of water in the emulsion template, the individual pores are connected through windows possibly forming a network of interconnected pores in the film. With interconnected pores, the passages and transport of water and other molecules are enabled through a film with the prospect to be used for delivery or barrier applications.

3.1.2. Bilayer Films. Formation of bilayer PHB films consisting of a porous and a dense backing layer was explored in order to obtain films with new degradation characteristics that could enable a directional transport of nutrients or drugs or provide additional mechanical strength to a porous film. A two-step method is in many cases the preferred choice since it offers a way of controlling the thickness and the morphology of this second layer in comparison to a spontaneously formed two-layered film. These film layers need to be well fused together with no gap or slip between the layers in order to give a mechanical support or directed drug delivery. The SEM image in Figure 3(a) shows a highly porous PHB layer which has been combined with an approximately $10 \mu \mathrm{m}$ thick PHB backing layer. The SEM image at high resolution (see Figure 3(b)) shows that the two layers have been joined and well fused together during film formation.

3.2. Permeability. There are many factors that affect the molecular diffusion of a permeant through a film, mainly the diffusion routes available in the polymer matrix but also the size and chemical properties of the permeant. A major factor determining the diffusion rate is the presence of pores or channels to pass through.

The permeability of a film is closely related to the diffusion of the solute or permeant. Fick's first law of diffusion is

$$
J=-D \frac{d c}{d z}
$$

where $J$ is the flux per unit area, $D$ is the diffusion coefficient, and $d c / d z$ is the concentration gradient of the permeant in $z$ direction. However, a film is rarely a homogenous system so the diffusion across a film should be replaced by the effective 


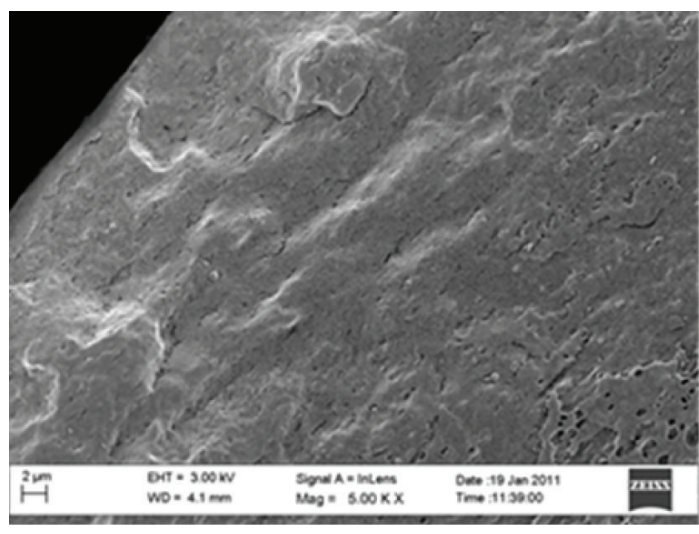

(a)

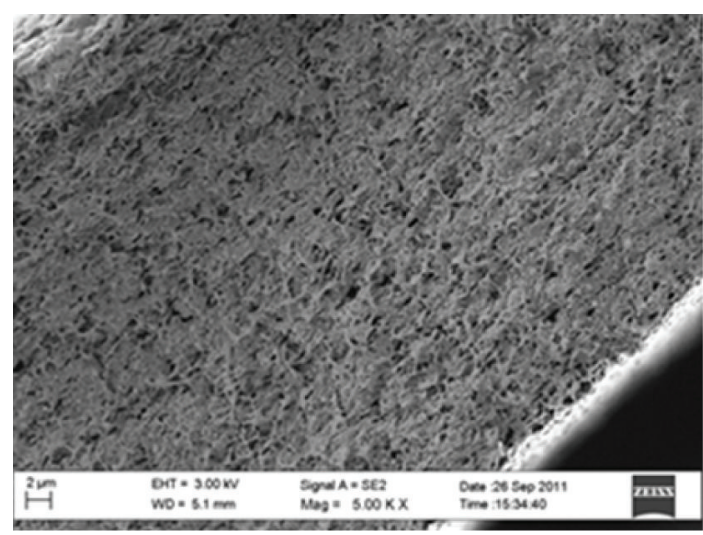

(c)

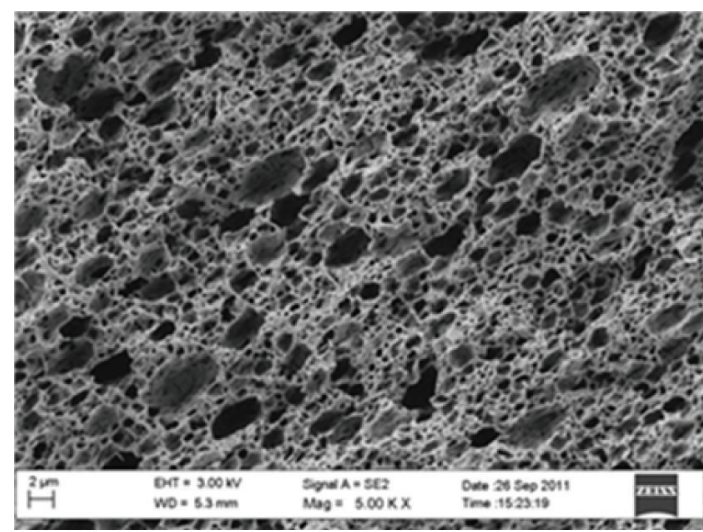

(e)

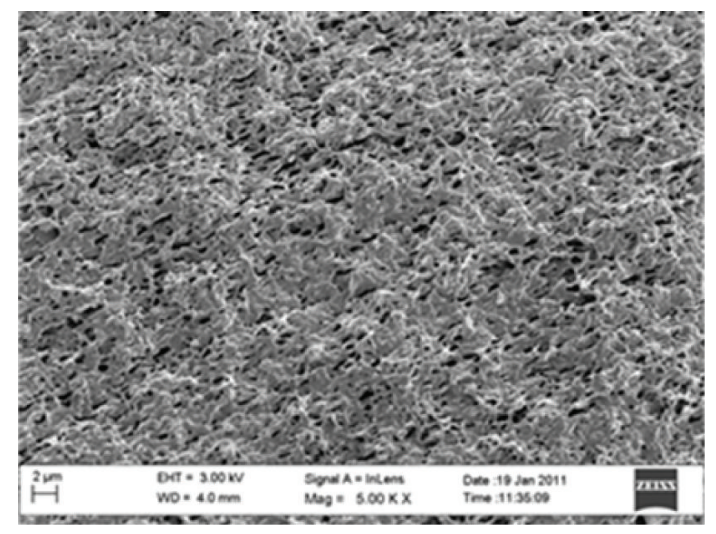

(b)

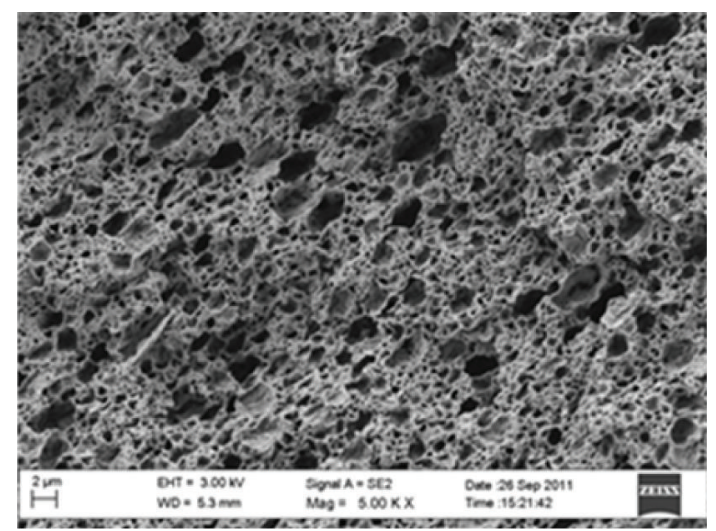

(d)

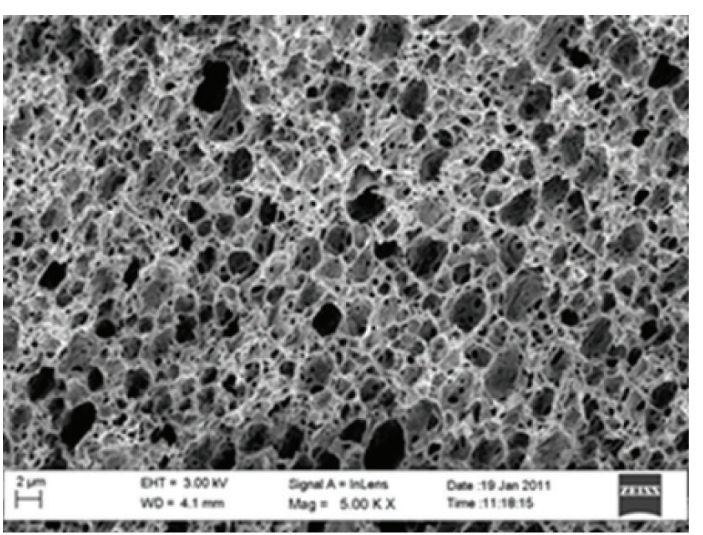

(f)

FIGURE 1: SEM images of PHB film cross sections made with (a) no emulsion template, (b) $3 \%$, (c) $6 \%$, (d) $8 \%$, (e) $9 \%$, and (f) $10 \%$ of water in the emulsion template. The lithium salt content of the water phase was $2.9 \%(\mathrm{w} / \mathrm{v})$ for all template emulsions. The scale bar is $2 \mu \mathrm{m}$ and the magnification is $\times 5 \mathrm{k}$. The picture was taken with horizontal detection mode.

diffusion coefficient $D_{e}$. The steady state diffusion can be written as

$$
J=-\frac{D_{e} A}{h}\left(c_{d}-c_{a}\right)
$$

where $D_{e}$ is the effective diffusion coefficient, $A$ is the area of diffusion, $h$ is the film thickness, and $c_{d}$ and $c_{a}$ are the concentrations of the permeant in the donor and acceptor compartments, respectively. The effective diffusion across a film may be written in terms of permeability as

$$
J=-P A\left(c_{d}-c_{a}\right),
$$

and by combining (3) and (2), the permeability becomes related to diffusion as follows:

$$
P=\frac{D_{e}}{h} .
$$




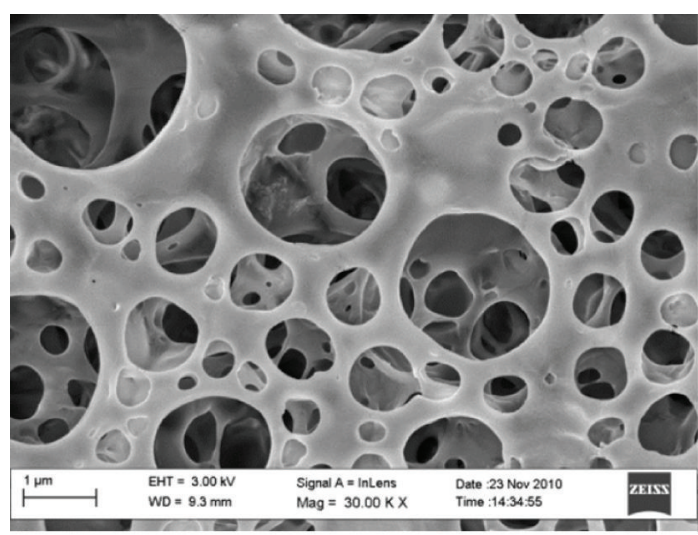

FIGURE 2: SEM image of the surface of a PHB film made with $10 \%$ of water in the emulsion template taken with in-lens detection mode. The scale bar is $1 \mu \mathrm{m}$ and the magnification is $\times 30 \mathrm{k}$.

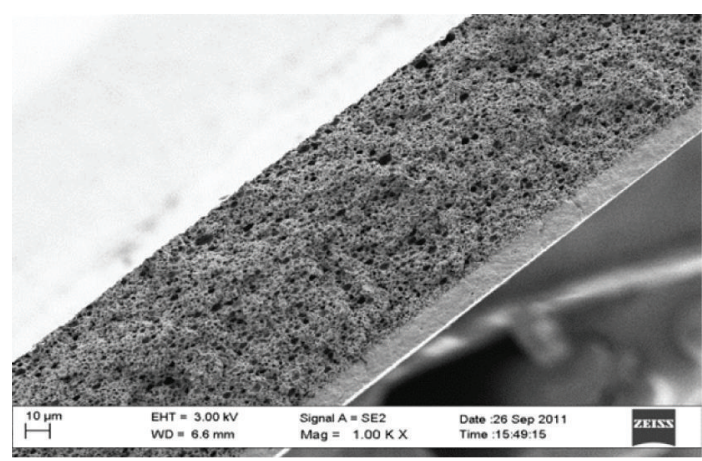

(a)

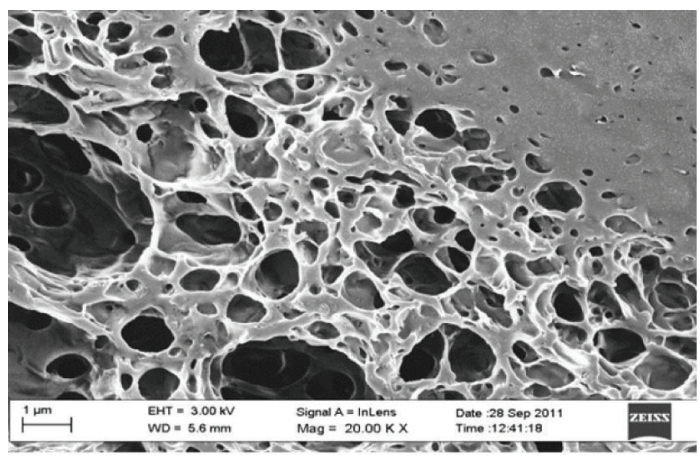

(b)

FIGURE 3: SEM image taken by in-lens detection mode of a bilayer PHB film combining a dense PHB layer with a porous layer made with $10 \%$ of water in the emulsion template showing (a) the whole cross section (scale bar $10 \mu \mathrm{m}$ and magnification $\times 1 \mathrm{k}$ ) and (b) the interface between the two layers at a higher magnification (scale bar $1 \mu \mathrm{m}$ and magnification $\times 20 \mathrm{k}$ ).

The water permeability of PHB films was determined by measuring the radioactivity of tritiated water in the acceptor chamber at specific time intervals as CPM (counts per minute). A representative plot of the increase in CPM with time is shown in Figure 4.

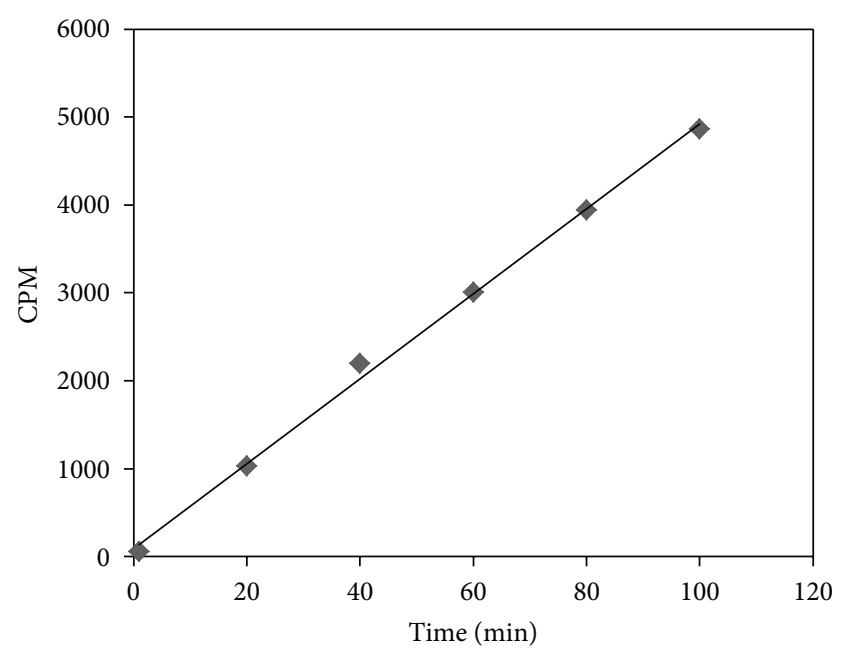

FIGURE 4: Exemplifying plot of the amount of radioactive water in CPM (counts per minute), having diffused across a single layer PHB film made from an emulsion template consisting of $10 \%$ of water and $2.9 \%(\mathrm{w} / \mathrm{v})$ of $\mathrm{Li}_{2} \mathrm{SO}_{4}$.

3.2.1. Water Permeability of Single and Bilayer Films. For a porous PHB film, water will mainly pass through the available pores and channels, not penetrating the polymer matrix, due to the inherent crystallinity and hydrophobicity of PHB [19]. Figure 5 shows the influence of pore forming water-droplet content in template emulsions on the permeability of porous PHB films. The results show that the water permeability of the films increased with increasing water content in the emulsion template. The permeability of nonporous PHB films is very low $\left(3.7 \times 10^{-13} \mathrm{~m}^{2} / \mathrm{s}\right)$ and the permeability of films made with 3 to $6 \%$ water in the emulsion template are in the same order of magnitude. The film made from $8 \%$ water has a permeability $28.4 \times 10^{-13} \mathrm{~m}^{2} / \mathrm{s}$ which is marginally higher. At lower water contents in the emulsion templates the pore forming droplets are scarce probably resulting in non-adjacent pores that are dispersed throughout the film. The water permeability is then largely determined by the low permeability of the dense $\mathrm{PHB}$ matrix. At higher water amounts $(9-10 \%)$ the permeability is increased by almost three orders of magnitude suggesting that the number of droplets is sufficient and they have come close enough during film formation to form interconnected pore structures or even channels in which the water will pass.

The permeability of double layered films was also determined. Figure 6 compares permeability data of a film combining a nonporous and a porous layer with the data of the respective layers. The dense layer comprises one-tenth of the total thickness of the bilayer film as was shown in Figure 3. The permeability of the bilayer has become equally low as the dense PHB film which means that the dense portion of the bilayer film fully determines the film permeability.

In summary, these data show that the bilayer film has equally low permeability as a dense PHB film and in addition enables unidirectional drug release or new degradation characteristics. 


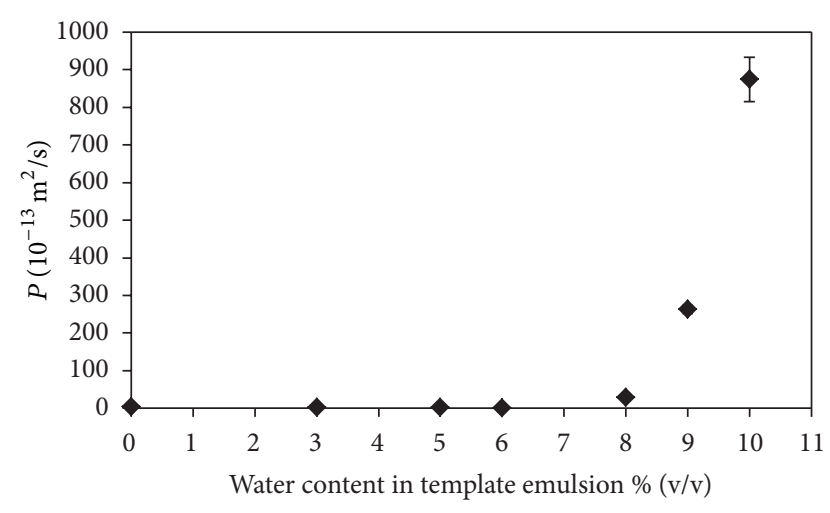

FIGURE 5: Plot of the water permeability of films casted from template emulsions with varying water content in the emulsion template. The permeability was normalized against the film thickness. Error bars indicate $\mathrm{min} / \mathrm{max}$ deviation $(n=2-3)$.

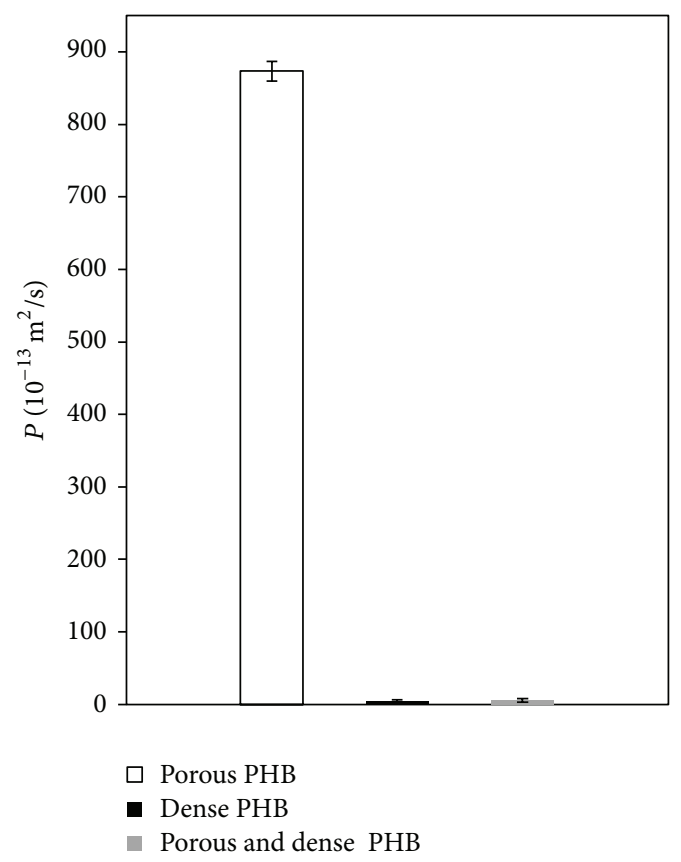

FIGURE 6: Water permeability of porous, dense (nonporous), and bilayer films casted from template emulsions with $10 \%(\mathrm{v} / \mathrm{v})$ of water and $2.9 \%(\mathrm{w} / \mathrm{v})$ of $\mathrm{Li}_{2} \mathrm{SO}_{4}$ in the emulsion template. The permeability was normalized against the film thickness. Error bars indicate $\min / \max$ deviation $(n=2-3)$.

3.2.2. Fine Tuning of Film Permeability. It has previously been shown by us that including lithium sulphate in the water phase is a way of facilitating the translation of template emulsion droplets into spherical pores which are evenly dispersed in the resulting PHB film [19]. In this study we have elaborated on the concept even further and varied the concentrations of lithium sulphate in the template emulsion with the highest water content to see how this influences the film morphology and if this would then influence the film permeability. The water permeability of films made from

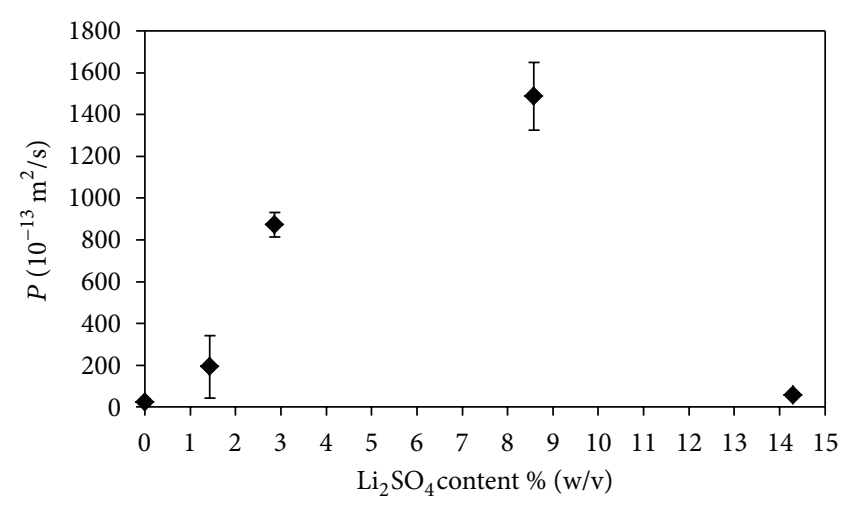

FIGURE 7: Plot of the water permeability for films casted from template emulsions with $10 \%$ of water and increasing $\mathrm{Li}_{2} \mathrm{SO}_{4}$ content in the water phase. The permeability was normalized against the film thickness. Error bars indicate $\mathrm{min} / \mathrm{max}$ deviation $(n=2-3)$.

template emulsions with various amounts of lithium sulphate included in the water phase is shown in Figure 7.

The film made from $0 \%$ salt in the template emulsion has a very low permeability of approximately $24 \times 10^{-13}\left(\mathrm{~m}^{2} / \mathrm{s}\right)$ as is seen in Figure 7. SEM images of this type of film discloses a highly porous film but with irregular pores, thick pore walls, and low number of windows between the pores (for SEM pictures see [19]). The permeability is comparable to that of films made from $0-8 \%$ water in the template emulsions. Also these films appear to have a low degree of interconnectivity between the pores as suggested by the SEM images shown in Figure 1. The results in Figure 7 show that the permeability of films made from template emulsion with $1.4-14.3 \%(\mathrm{w} / \mathrm{v})$ lithium salt varies a 30 -fold with changed salt content of the template emulsion. The $\mathrm{PHB}$ film with the highest permeability $(8.7 \%(\mathrm{w} / \mathrm{v})$ lithium salt) is comparable in terms of permeability to ethyl cellulose (EC) films made porous with $30 \%$ water soluble hydroxypropyl cellulose [21]. These films have a permeability of $250^{-12}\left(\mathrm{~m}^{2} / \mathrm{s}\right)$, yet a porosity of about $30 \%$. The high permeability may be explained by the material morphology. The EC film is made by spray drying and the material is formed during spinoidal decomposition resulting in thin, continuous channels through the film. The morphology of highly porous $\mathrm{PHB}$ films is slightly different compared to EC films, with larger pores which are connected through small windows. The interconnectivity is largely dependent on the window formation and pores may to some extent exist as discrete nonconnected pores which would then result in a lower water permeability compared to the EC films.

The diverse water permeability of PHB films made from emulsion templates with varied salt content is most likely a result of differences in the film morphology and window formation between pores in film. The low permeable PHB films would reasonably then have a lower degree of porosity or interconnectivity between the pores than the high permeable PHB films. The SEM images of these films however are very similar, showing highly porous films with windows of approximately $0.2-0.5 \mu \mathrm{m}$, which might be connecting 
TABLE 1: The porosity of films casted from template emulsions with a variation in water volume and lithium salt content $(n=3)$.

\begin{tabular}{lcc}
\hline $\begin{array}{l}\text { Water content } \\
\%(\mathrm{v} / \mathrm{v})\end{array}$ & $\begin{array}{c}\mathrm{Li}_{2} \mathrm{SO}_{4} \text { content } \\
\%(\mathrm{w} / \mathrm{v})\end{array}$ & $\begin{array}{c}\text { Porosity } \\
\%(\mathrm{w} / \mathrm{w})\end{array}$ \\
\hline 0 & 0 & $1 \pm 0$ \\
5 & 2.9 & $16 \pm 14$ \\
6 & 2.9 & $17 \pm 14$ \\
8 & 2.9 & $39 \pm 7$ \\
9 & 2.9 & $47 \pm 6$ \\
10 & 2.9 & $52 \pm 3$ \\
10 & 0 & $51 \pm 3$ \\
10 & 1.4 & $51 \pm 6$ \\
10 & 2.9 & $52 \pm 3$ \\
10 & 8.7 & $57 \pm 7$ \\
10 & 14.3 & $45 \pm 3$ \\
\hline
\end{tabular}

adjacent pores. The SEM images however cannot disclose any evident variation in porosity and pore interconnectivity that would account for the diversity in permeability of the films.

3.3. Porosity. The degree of porosity of films made from template emulsions with varying amount of water or lithium salt in the water phase was determined in order to see how well the degree of porosity correlates with the respective film permeability. The porosity $(\Phi)$ of a material can be estimated using the apparent density and true density according to (5). The porosity was determined for all films by calculating the apparent density of the film $\left(\rho_{a}\right)$ and comparing it to the true density $\left(\rho_{t}\right)$ of PHB which is $1.22 \mathrm{~g} \mathrm{~cm}^{-3}$ according to the literature [22]. The $\rho_{a}$ was derived from the weight and dimensions of the porous PHB films:

$$
\Phi=1-\frac{\rho_{a}}{\rho_{t}}
$$

Table 1 shows the porosity of pure PHB films and films made from emulsion templates with various amounts of water and lithium salt content.

The solvent casted pure PHB film made without emulsion template showed almost no porosity. The film porosity increases with the amount of water in the emulsion template. The porosities of films made from 5 and $6 \%(\mathrm{v} / \mathrm{v})$ water in the template emulsion are about $16-17 \%$. The quite large standard deviation for the 5 and $6 \%(\mathrm{v} / \mathrm{v})$ films is probably an effect of the low viscosity of the sample which results in a less stabile template emulsion and variations in the template emulsion which is translated to the resulting film. The film morphology of these films is somewhat different than the other porous films made from emulsion templates. Films made from template emulsions with $8-10 \%(\mathrm{v} / \mathrm{v})$ water showed an increase in degree of porosity from $47 \%$ up to $52 \%$ with increased concentration. However, films made from template emulsions of $10 \%(\mathrm{v} / \mathrm{v})$ water show little effect of varied lithium salt content in the water phase on the degree of porosity (45-57\%). A film made from a lithium salt deficient template emulsion results in a film with as high as $51 \%$ porosity. The results from porosity analysis show that the concentration of salt in the template emulsions has little or no effect on the porosity of the films. However it seems that there is a relationship between the amount of water in the emulsion template and the degree of porosity of the tested films.

Taken all data into account, the SEM images and the porosity analysis provide information about the film morphology and porosity. However it is essential to perform permeability analysis for these kinds of films to get an understanding of the pore interconnectivity.

\section{Conclusions}

In this study it was shown that it is possible to make highly porous PHB films with a tunable permeability by changing the composition of the emulsion template. The results show that the water permeability of the films can be varied by modifying the film morphology as a result of changing the lithium salt content of the template emulsion. The results further suggest that the water permeability of the films tested in this study is not directly correlated to the degree of porosity but to the extent of pore interconnectivity. Furthermore, we report on the formation of bilayer PHB films consisting of a porous and a dense backing layer which were well fused together with no visible gap between the layers. The permeability analysis showed that the bilayer film has equally low permeability as the dense PHB film. These porous PHB films of single and bilayer type are interesting in a future perspective for the use as temporary barriers or directional transport of nutrients or drugs. Possible future works would include the design and production of multilayered films with drugs or bioactive molecules incorporated in or between the different layers, investigate the degradation profile, and perform permeability studies using drugs or larger biomolecules.

\section{Conflict of Interests}

The authors declare that there is no conflict of interests regarding the publication of this paper.

\section{Acknowledgments}

The authors thank Anders Mårtensson at the Department of Chemical and Biological Engineering, Chalmers University of Technology, for his help with the SEM image analysis. This work was performed by financial support from the VINN Excellence Centre SuMo Biomaterials (Supermolecular Biomaterials-structure dynamics and properties) and from Chalmers Area of Advance, Materials Science, Chalmers University of Technology.

\section{References}

[1] T. Saito, K. Tomita, K. Juni, and K. Ooba, "In vivo and in vitro degradation of poly(3-hydroxybutyrate) in rat," Biomaterials, vol. 12, no. 3, pp. 309-312, 1991. 
[2] S. Gogolewski, M. Jovanovic, S. M. Perren, J. G. Dillon, and M. K. Hughes, "Tissue response and in vivo degradation of selected polyhydroxyacids: polylactides (PLA), poly(3hydroxybutyrate) (PHB), and poly(3- hydroxybutyrate-co-3hydroxyvalerate) (PHB/VA)," Journal of Biomedical Materials Research, vol. 27, no. 9, pp. 1135-1148, 1993.

[3] T. Malm, S. Bowald, A. Bylock, and C. Busch, "Prevention of postoperative pericardial adhesions by closure of the pericardium with absorbable polymer patches: an experimental study," The Journal of Thoracic and Cardiovascular Surgery, vol. 104, no. 3, pp. 600-607, 1992.

[4] C. Doyle, E. T. Tanner, and W. Bonfield, "In vitro and in vivo evaluation of polyhydroxybutyrate and of polyhydroxybutyrate reinforced with hydroxyapatite," Biomaterials, vol. 12, no. 9, pp. 841-847, 1991.

[5] T. Malm, S. Bowald, A. Bylock, C. Busch, and T. Saldeen, "Enlargement of the right ventricular outflow tract and the pulmonary artery with a new biodegradable patch in transannular position," European Surgical Research, vol. 26, no. 5, pp. 298308, 1994.

[6] S. Y. Lee, "Bacterial polyhydroxyalkanoates," Biotechnology and Bioengineering, vol. 49, pp. 1-14, 1996.

[7] Y. Doi, Y. Kanesawa, Y. Kawaguchi, and M. Kunioka, "Hydrolytic degradation of microbial poly(hydroxyalkanoates)," Die Makromolekulare Chemie, Rapid Communications, vol. 10, pp. 227-230, 1989.

[8] T. Freier, C. Kunze, C. Nischan et al., "In vitro and in vivo degradation studies for development of a biodegradable patch based on poly(3-hydroxybutyrate)," Biomaterials, vol. 23, no. 13, pp. 2649-2657, 2002.

[9] M. Åberg, C. Ljungberg, E. Edin et al., "Clinical evaluation of a resorbable wrap-around implant as an alternative to nerve repair: a prospective, assessor-blinded, randomised clinical study of sensory, motor and functional recovery after peripheral nerve repair," Journal of Plastic, Reconstructive \& Aesthetic Surgery, vol. 62, no. 11, pp. 1503-1509, 2009.

[10] P.-N. Mohanna, R. C. Young, M. Wiberg, and G. Terenghi, "A composite pol-hydroxybutyrate-glial growth factor conduit for long nerve gap repairs," Journal of Anatomy, vol. 203, no. 6, pp. 553-565, 2003.

[11] C. Rentsch, B. Rentsch, A. Breier et al., "Evaluation of the osteogenic potential and vascularization of 3D poly(3)hydroxybutyrate scaffolds subcutaneously implanted in nude rats," Journal of Biomedical Materials Research A, vol. 92, no. 1, pp. 185-195, 2010.

[12] L. Lu, S. J. Peter, M. D. Lyman et al., "In vitro and in vivo degradation of porous poly(DL-lactic-co-glycolic acid) foams," Biomaterials, vol. 21, no. 18, pp. 1837-1845, 2000.

[13] A. G. Mikos, G. Sarakinos, S. M. Leite, J. P. Vacanti, and R. Langer, "Laminated three-dimensional biodegradable foams for use in tissue engineering," Biomaterials, vol. 14, no. 5, pp. 323330, 1993.

[14] C. Vaquette, C. Frochot, R. Rahouadj, and X. Wang, "An innovative method to obtain porous PLLA scaffolds with highly spherical and interconnected pores," Journal of Biomedical Materials Research B, vol. 86, no. 1, pp. 9-17, 2008.

[15] H. Lo, M. S. Ponticiello, and K. W. Leong, "Fabrication of controlled release biodegradable foams by phase separation," Tissue Engineering, vol. 1, pp. 15-28, 1995.

[16] A. C. Richards Grayson, I. S. Choi, B. M. Tyler et al., "Multipulse drug delivery from a resorbable polymeric microchip device," Nature Materials, vol. 2, no. 11, pp. 767-772, 2003.
[17] W. H. Ryu, M. Vyakarnam, R. S. Greco, F. B. Prinz, and R. J. Fasching, "Fabrication of multi-layered biodegradable drug delivery device based on micro-structuring of PLGA polymers," Biomedical Microdevices, vol. 9, no. 6, pp. 845-853, 2007.

[18] A. Bergstrand, S. Uppström, and A. Larsson, "Porous biodegradable coatings for drug delivery," European Cells and Materials, vol. 21, supplement 1, p. 24, 2011.

[19] A. Bergstrand, H. Andersson, J. Cramby, K. Sott, and A. Larsson, "Preparation of porous poly (3-hydroxybutyrate) films by water-droplet templating," Journal of Biomaterials and Nanobiotechnology, vol. 3, pp. 431-439, 2012.

[20] J. Hjartstam and T. Hjertberg, "Studies of the water permeability and mechanical properties of a film made of an ethyl celluloseethanol-water ternary mixture," Journal of Applied Polymer Science, vol. 74, pp. 2056-2062, 1999.

[21] H. Andersson, J. Hjartstam, M. Stading, C. von Corswant, and A. Larsson, "Effects of molecular weight on permeability and microstructure of mixed ethyl-hydroxypropyl-cellulose films," European Journal of Pharmaceutical Sciences, vol. 48, pp. 240248, 2013.

[22] C. Pedros-Alio, J. Mas, and R. Guerrero, "The influence of poly$\beta$-hydroxybutyrate accumulation on cell volume and buoyant density in Alcaligenes eutrophus," Archives of Microbiology, vol. 143, no. 2, pp. 178-184, 1985. 

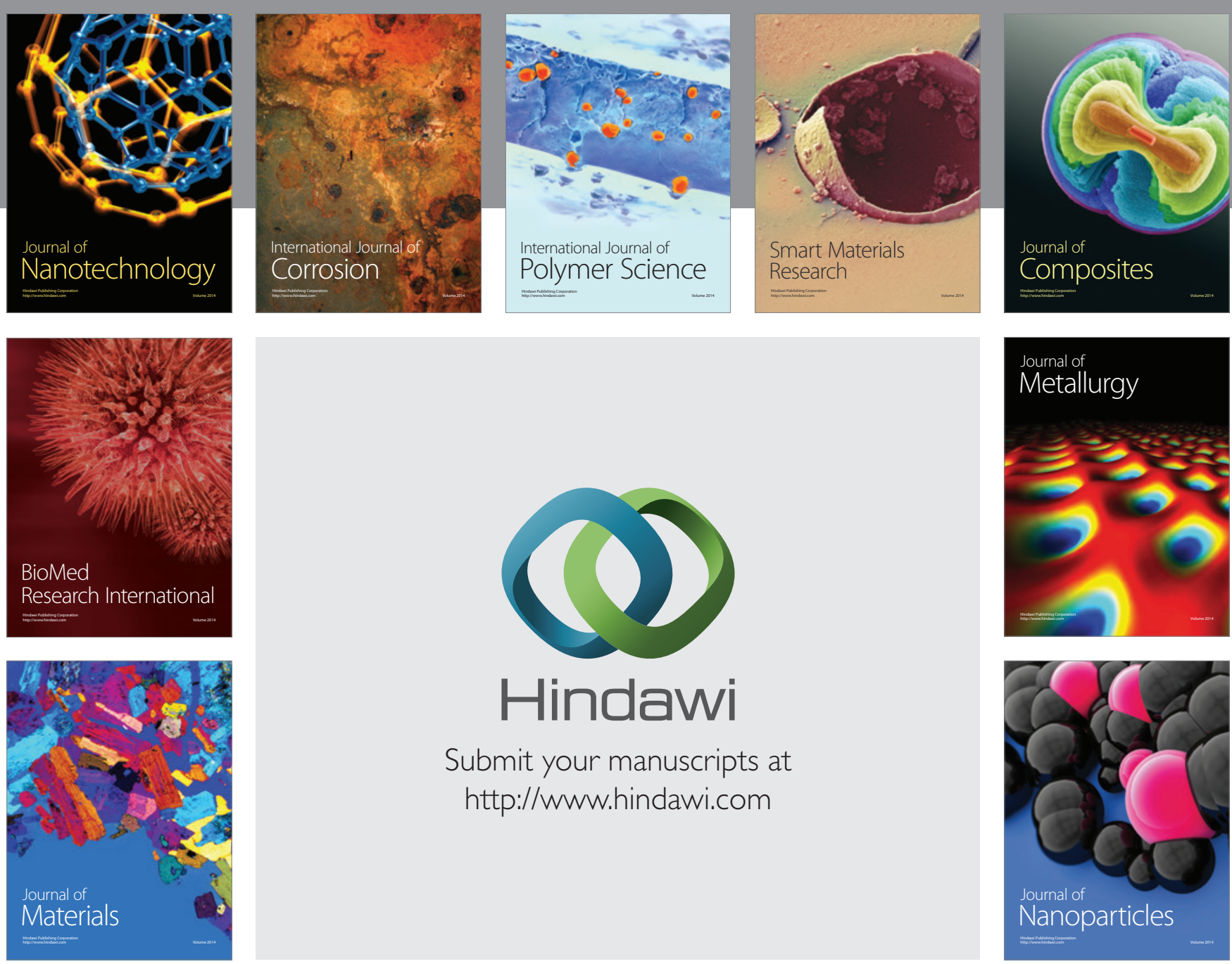

Submit your manuscripts at http://www.hindawi.com
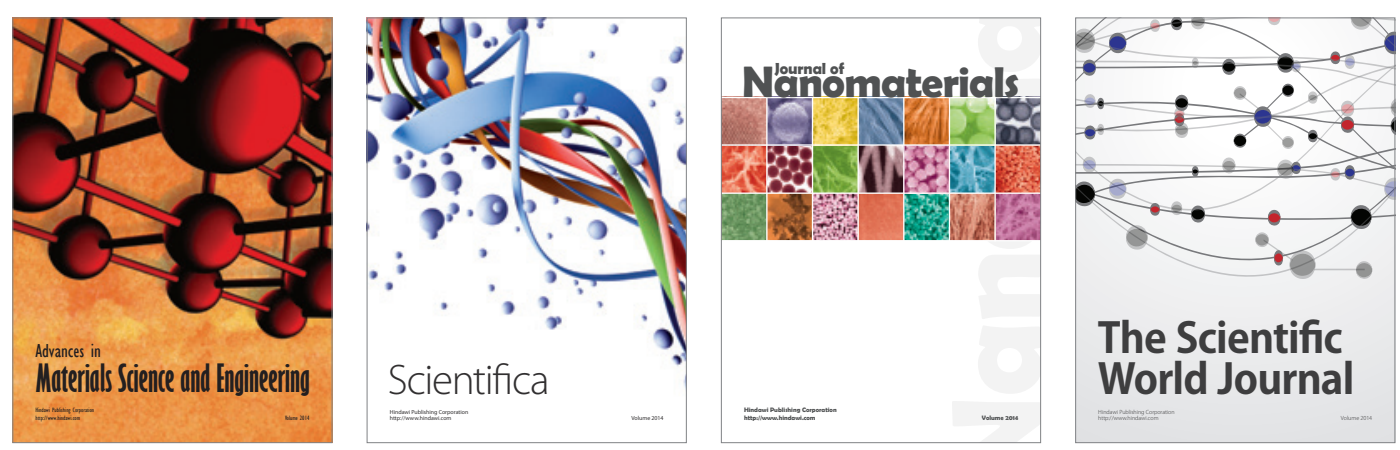

\section{The Scientific World Journal}
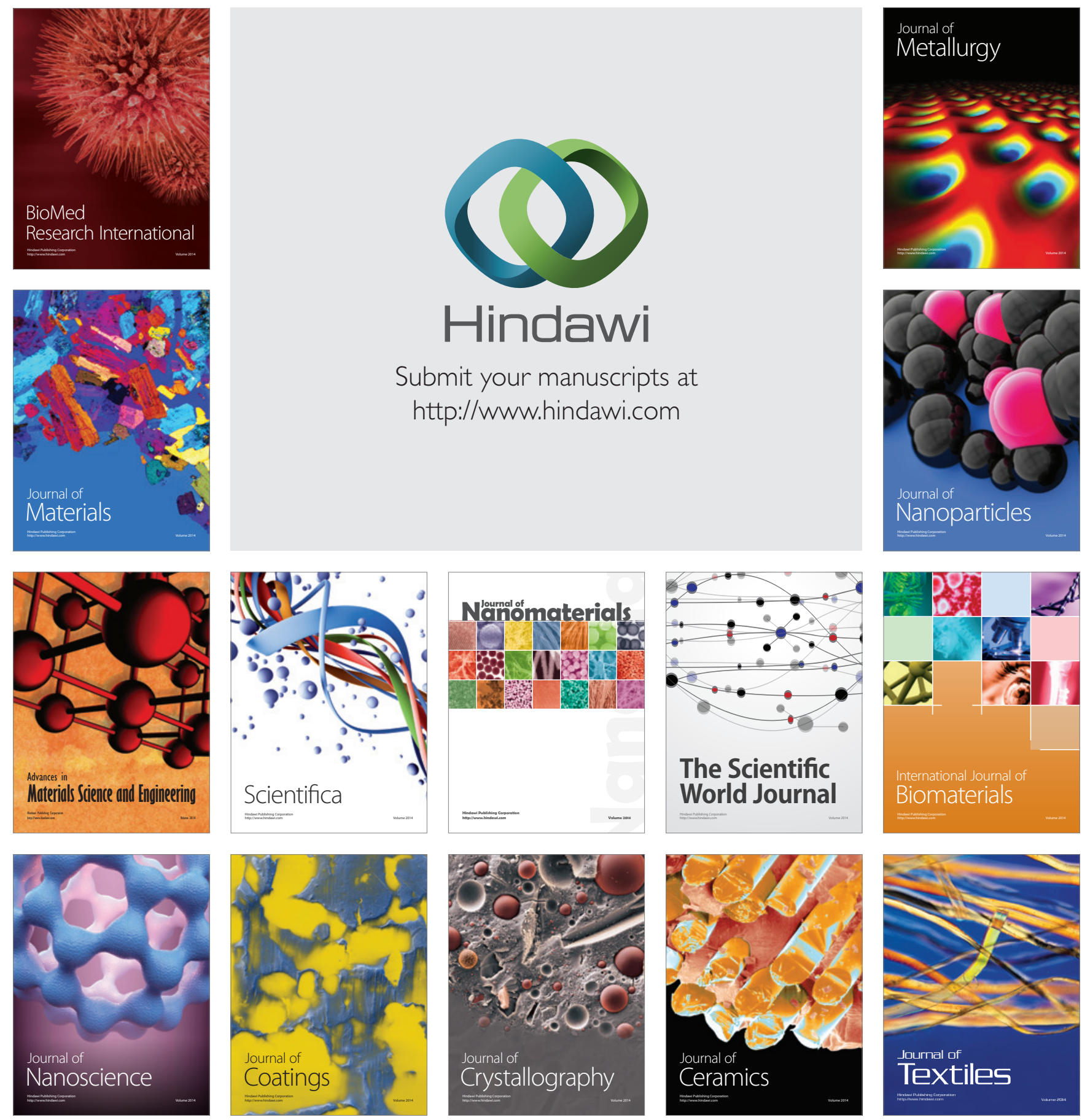